\title{
The interaction between androgen receptor and semenogelin I: a synthetic LxxLL peptide antagonist inhibits the growth of prostate cancer cells
}

\author{
Peng Li $i^{1,2,6}$, Jinbo Chen ${ }^{3,4,6}$, Eiji Kashiwagi ${ }^{1,2,6}$, Taichi Mizushima1,2,3,4,6, Bin Han ${ }^{1,2}$, Satoshi Inoue ${ }^{1,2,3,4}$, \\ Hiroki Ide ${ }^{1,2}$, Koji Izumi ${ }^{3}$ and Hiroshi Miyamoto*,1,2,3,4,5 \\ ${ }^{1}$ Department of Pathology, Johns Hopkins University School of Medicine, Baltimore, MD 21287, USA; ${ }^{2}$ James Buchanan Brady \\ Urological Institute, Johns Hopkins University School of Medicine, Baltimore, MD 21287, USA; ${ }^{3}$ Department of Pathology \& \\ Laboratory Medicine, University of Rochester Medical Center, Rochester, NY 14642, USA; ${ }^{4}$ James P. Wilmot Cancer Institute, \\ University of Rochester Medical Center, Rochester, NY 14642, USA and ${ }^{5}$ Department of Urology, University of Rochester Medical \\ Center, Rochester, NY 14642, USA
}

Background: We previously demonstrated that a seminal plasma protein, semenogelin I (Sgl), functioned as an androgen receptor (AR) coactivator. Meanwhile, several short sequence motifs in AR coregulators, such as $L x \times L L(L=$ leucine), have been shown to mediate specific interactions with AR.

Methods: We investigated the role of the LxxLL motif within Sgl in the interactions with AR and cell growth in prostate cancer lines in vitro.

Results: A full-length Sgl with mutations in the motif (i.e., LxxAA; A=alanine) failed to significantly increase cell proliferation/ migration as well as androgen-mediated AR transcription. Co-immunoprecipitation showed no physical interactions between AR and the mutant Sgl. In addition, transfection of an 18-amino acid peptide of Sgl containing LxxLL, but not LxxAA, resulted in considerable reduction in cell growth and prostate-specific antigen expression in LNCaP and C4-2 lines.

Conclusions: The LxxLL motif of Sgl could be a novel therapeutic target for both androgen-sensitive and castration-resistant prostate cancers.

Prostate cancer remains one of the most common causes of death in men worldwide (Torre et al, 2015). Although hormonal manipulation plays a critical role in the management of advanced hormone-naïve prostate cancer, most of these patients eventually develop castration-resistant disease for which treatment options are limited. Importantly, the activity of androgen receptor (AR), a member of the nuclear receptor superfamily, is well known to correlate with the growth of even castration-resistant prostate cancer (CRPC) (Coutinho et al, 2016). Accordingly, molecules that interact with $\mathrm{AR}$ and activate or inactivate its activity are potential therapeutic targets for both hormone-dependent and castrationresistant diseases. Indeed, previous studies have demonstrated that several short sequence motifs in these coregulators, such as LxxLL (where $\mathrm{L}$ is leucine and $\mathrm{x}$ is any amino acid), are critical for

\footnotetext{
*Correspondence: Dr H Miyamoto; E-mail: hiroshi_miyamoto@urmc.rochester.edu

${ }^{6}$ These authors contributed equally to this work.
}

Received 8 June 2017; revised 12 October 2017; accepted 13 October 2017; published online 14 November 2017

(C) 2018 Cancer Research UK. All rights reserved 0007-0920/18 
mediating specific interactions with AR (Heery et al, 1997; van de Wijngaart et al, 2012), and disruption of AR-coactivator interactions is expected to reduce AR-mediated transcriptional activity.

Semenogelins constitute the major structural proteins in human semen and are known to contribute to, upon binding to $\mathrm{Zn}^{2+}$ from the prostate, semen coagulation (Jonsson et al, 2006). After ejaculation, semenogelins are hydrolyzed by prostate-specific antigen (PSA), resulting in semen liquefaction and release of the entrapped spermatozoa (Lilja et al, 1987). Our immunohistochemical studies in prostate cancer tissue specimens showed that the levels of nuclear semenogelin I (SgI) expression was significantly elevated in prostatic carcinoma, compared with non-neoplastic prostatic epithelium or high-grade prostatic intraepithelial neoplasia, and that its overexpression in carcinoma strongly correlated with the risk of biochemical recurrence after radical prostatectomy (Canacci et al, 2011; Izumi et al, 2012). We have additionally demonstrated, using cell line models, that SgI functions as an AR coactivator only in the presence of $\mathrm{Zn}^{2+}$ and thereby enhances the androgen-mediated growth of prostate cancer cells (Canacci et al, 2011; Ishiguro et al, 2015). These preclinical findings may also explain why prostate cancer tissue contains high levels (400$800 \mu \mathrm{M}$ (Costello and Franklin, 2006)) of zinc that is highly cytotoxic (e.g. $100 \mu \mathrm{m}$ in PC3 culture, $250 \mu \mathrm{m}$ in LNCaP culture (Liang et al, 1999; Untergasser et al, 2000)).

There is a LxxLL motif in SgI protein, but no other motifs shown to mediate $\mathrm{AR}$-coregulator interactions are present. We therefore anticipated that the LxxLL motif was responsible for mediating $\mathrm{AR}-\mathrm{SgI}$ interactions. In the current study, we aim to investigate the role of this motif present in SgI in the interactions with AR in prostate cancer cells as well as tumour growth.

\section{MATERIALS AND METHODS}

Plasmids and peptides. The entire coding region of SgI amplified using Phusion-High Fidelity DNA polymerase (Thermo Fisher Scientific, Waltham, MA, USA) was subcloned into pSG5 (Canacci et al, 2011). A pSG5 construct containing a full-length SgI with mutations in the LxxLL motif (LxxAA where $\mathrm{A}$ is alanine; Figure 1A) generated by a site-directed mutagenesis method (GeneArt Site-Directed Mutagenesis System, Invitrogen, Carlsbad, CA, USA) was also used. pSG5-AR, pGL3-MMTV-luciferase, and pRL-TK have been described in our previous studies (Miyamoto et al, 2005; Zheng et al, 2015). In addition, 18-amino acid peptides of SgI containing the LxxLL motif (wild-type: MKPNIIFVLSLLLILEKQ) or the LxxAA motif (mutant: MKPNIIFVLSLAAILEKQ) were obtained from GenScript (Piscataway, NJ, USA).

Cell lines and chemicals. Human prostate cancer cell lines (PC3, LNCaP, and C4-2) recently authenticated by the institutional core facility were maintained in RPMI 1640 (Mediatech, Manassas, VA, USA) supplemented with $10 \%$ foetal bovine serum (FBS) at $37^{\circ} \mathrm{C}$ in a humidified atmosphere of $5 \% \mathrm{CO}_{2}$. The plasmids and peptides were transfected into the cell lines, using transfection reagents, Lipofectamine 3000 (Thermo Fisher Scientific) and Xfect (Clontech, Mountain View, CA, USA), respectively. Anti-AR (N-20), anti-SgI (E-15), and anti-GAPDH (6C5) antibodies were purchased from Santa Cruz Biotechnology (Dallas, TX, USA). An antiPSA antibody (A0562) was purchased from Dako (Carpinteria, CA, USA). Dihydrotestosterone (DHT) and $\mathrm{ZnCl}_{2}$ were from Sigma-Aldrich (St Louis, MO, USA) and Alfa Aesar (Haverhill, MA, USA), respectively.

Western blot. Equal amounts of protein obtained from cell extracts were separated in $10 \%$ sodium dodecyl sulfate (SDS)polyacrylamide gel electrophoresis (PAGE), transferred to polyvinylidene difluoride membrane (Immun-Blot PVDF Membrane, Bio-Rad, Hercules, CA, USA) electronically, blocked, and

A

\section{Wild-type (WT) ctt tccetgctcctc LxxLL Mutant (MT) ct t tccetggCcgCC LxxAA}

B
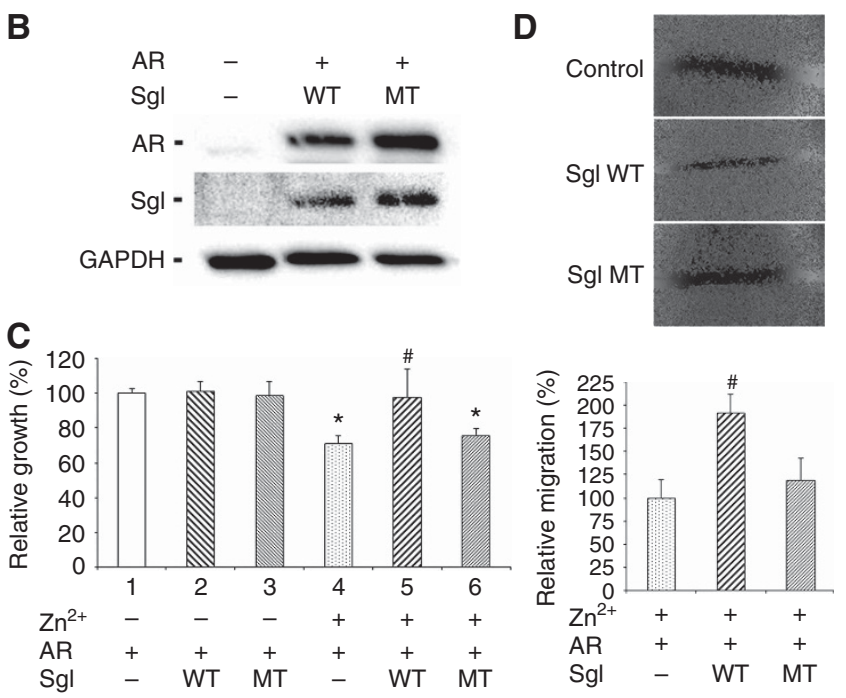

Figure 1. The effects of wild-type (WT) vs mutant (MT) Sgl overexpression on the growth of prostate cancer cells. (A) Sequencing of wild-type Sgl containing the LxxLL motif and a mutant Sgl containing LxxAA. (B) Total proteins extracted from PC3 cells transfected with pSG5, pSG5-AR, and/or pSG5-Sgl were analysed on western blots, using an antibody to AR (110 kDa), Sgl (52 kDa), or GAPDH (37 kDa). (C) PC3 cells were transfected with pSG5, pSG5-AR, and/or pSG5-Sgl, and subsequently cultured in medium supplemented with $10 \%$ normal FBS in the presence or absence of $100 \mu \mathrm{m}$ zinc for $96 \mathrm{~h}$. Cell viability assayed with MTT is presented relative to cell number in the control line without zinc treatment (lane 1; set as 100\%). Each value represents the mean + s.d. of three independent determinations. (D) PC3 cells transfected with pSG5, pSG5-AR, and/or pSG5-Sgl, and grown to confluence were gently scratched, and the wound area was measured after 24-h culture. The migration determined by the rate of cells filling the wound area is presented relative to that of control line (first lane; set as $100 \%$ ). Each value represents the mean + s.d. of three independent determinations. ${ }^{\star} P<0.05$ (vs control cells without zinc treatment).

${ }^{\#} P<0.05$ (vs control cells with zinc treatment).

incubated with a specific primary antibody. The membrane was then incubated with a secondary antibody (anti-mouse or antirabbit IgG-HRP, Cell Signaling Technology, Danvers, MA, USA), and specific signals were detected, using a chemiluminescent substrate kit (Bio-Rad).

Cell proliferation. Cells $\left(2-3 \times 10^{3}\right.$ per well $)$ seeded in 96-well tissue culture plates were transfected with the plasmids or peptides and incubated. After $72-96 \mathrm{~h}$ of treatment, $10 \mu \mathrm{l}$ methylthiazolyldiphenyl-tetrazolium bromide (MTT) stock solution $\left(5 \mathrm{mg} \mathrm{ml}^{-1}\right.$; Sigma-Aldrich) was added to each well with $100 \mu$ l of medium for $4 \mathrm{~h}$ at $37^{\circ} \mathrm{C}$. The medium was replaced with $100 \mu \mathrm{l}$ dimethyl sulfoxide, followed by incubation for $5 \mathrm{~min}$ at room temperature. The absorbance at a wavelength of $570 \mathrm{~nm}$ with background subtraction at $655 \mathrm{~nm}$ was then measured.

Cell migration. Cells transfected with the plasmids at a density of $90-100 \%$ confluence in 12-well plates were scratched manually with a sterile $200 \mu \mathrm{l}$ plastic pipette tip, cultured for $24 \mathrm{~h}$, fixed with methanol, and stained with $0.1 \%$ crystal violet. The width of the wound area was quantitated, using ImageJ software (National Institutes of Health, Bethesda, MD, USA).

Reporter gene assay. Cells at a density of $50-70 \%$ confluence in 24-well plates were transfected with an androgen response 
element-reporter (MMTV-luciferase), pSG5-AR, either pSG5 or pSG5-SgI (wild-type or mutant), and a control reporter (pRL-TK). After $6 \mathrm{~h}$ of transfection, the cells were treated with zinc and/or DHT for $24 \mathrm{~h}$, and the luciferase activity was determined in the cell lysates, using a Dual-Luciferase Reporter Assay kit (Promega, Madison, WI, USA).

Co-immunoprecipitation. The lysates from cells transfected with pSG5-AR and pSG5-SgI (wild-type or mutant) were incubated with the anti-AR antibody for $16 \mathrm{~h}$ at $4{ }^{\circ} \mathrm{C}$ with agitation. Protein $\mathrm{A} / \mathrm{G}$-agarose beads were then added, and binding proteins were eluted. The eluted proteins were analysed by western blotting with an anti-AR or anti-SgI antibody.

Statistical analysis. Student's $t$-test was used to analyse differences in variables with a continuous distribution. $P$ values less than 0.05 were considered statistically significant.

\section{RESULTS}

A SgI with mutations in the LxxLL motif and prostate cancer cell growth. To compare the effects of wild-type SgI with the LxxLL motif $v s$ a mutant SgI without the LxxLL motif on ARmediated prostate cancer cell growth, AR-negative/SgI-negative (Canacci et al, 2011) PC3 cells were transfected with AR and either empty vector, wild-type SgI, or mutant SgI (Figure 1B). We then performed MTT assay and scratch wound healing assay to determine cell viability and migration, respectively. In accordance with our previous findings (Canacci et al, 2011; Ishiguro et al, 2015), in MTT assay (Figure 1C), zinc treatment (100 $\mu \mathrm{M})$ significantly inhibited the growth of control cells (lanes $1 v s 4$ ) and, only in the presence of zinc, wild-type SgI significantly increased the cell growth (lanes $4 v s$ 5). Thus, SgI overexpression resulted in protection from zinc cytotoxicity (lanes 2 vs 5). By contrast, a mutant SgI did not significantly change the growth rate in the absence (lanes $1 v s$ 3) or presence (lanes $4 v s$ 6) of zinc, compared with respective controls. Similarly, in wound healing assay in the presence of zinc, wild-type SgI (1.92-fold, $P=0.01)$ significantly induced the migration of cells, whereas the mutant SgI (1.19-fold, $P=0.33$ ) failed to do it (Figure 1D).

Interaction between AR and a SgI with mutations in the LxxLL motif. To assess the effects of a mutant SgI without the LxxLL motif on androgen-mediated AR transactivation, luciferase activity was first determined in PC3 cells transfected with AR and an androgen response element-reporter plasmid, and subsequently treated with zinc and DHT. DHT increased AR transcription by 7.59-fold, as compared with mock treatment, and wild-type SgI overexpression further enhanced DHT-mediated AR transactivation by 2.73-fold (Figure 2A). However, mutant SgI showed no significant effect on DHT-induced AR transcription (1.07-fold).

We have previously shown that wild-type SgI can physically interact with $\mathrm{AR}$ in prostate cancer cells (Ishiguro et al, 2015). To further investigate the interaction between AR and the mutant SgI, co-immunoprecipitation assay, using lysates of PC3 cells with transfection of AR and SgI, was performed. Using an anti-AR antibody, we precipitated the AR-binding protein complex in the protein lysate. Western blotting then proved that AR was able to interact with wild-type SgI, but not with mutant SgI (Figure 2B).

SgI peptide containing the LxxLL motif and prostate cancer cell growth. We next determined whether SgI peptides with or without the LxxLL motif affect the cell growth of AR-positive androgen-sensitive prostate cancer LNCaP line and its androgenindependent subline C4-2. SgI was shown to be expressed in LNCaP (Canacci et al, 2011) and C4-2 (data not shown), which was further induced by zinc treatment. To selectively interfere with the interaction between endogenous $\mathrm{AR}$ and SgI, SgI peptides

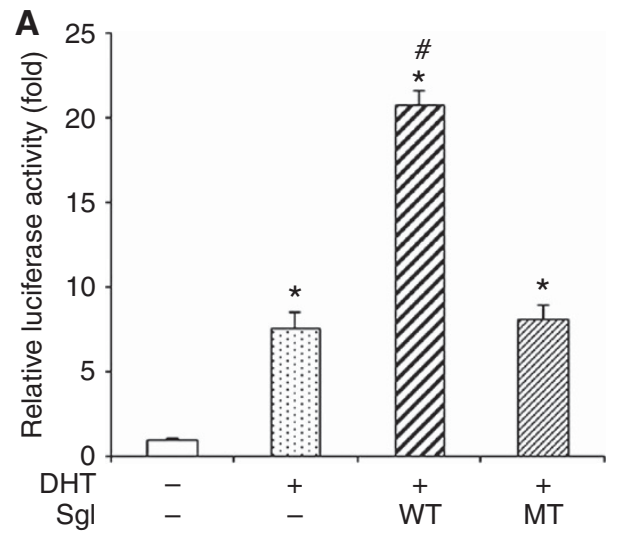

B

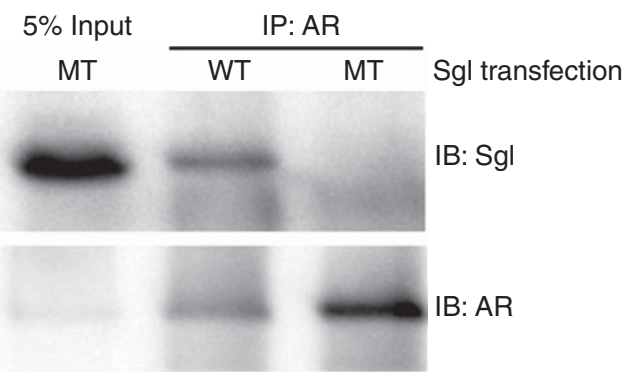

Figure 2. The effects of wild-type (WT) vs mutant (MT) Sgl on AR activity in prostate cancer cells. (A) PC3 cells were co-transfected with pSG5-AR (5 ng), pSG5 or pSG5-Sgl (250 ng), MMTV-luciferase (250 ng), and PRL-TK $(2.5 \mathrm{ng})$, and cultured in phenol red-free medium supplemented with $5 \%$ charcoal-stripped FBS along with zinc $(100 \mu \mathrm{m})$ and either mock (ethanol) or $10 \mathrm{nM} \mathrm{DHT}$ for $24 \mathrm{~h}$. The luciferase activity is presented relative to that of mock treatment (first lane; set as onefold). Each value represents the mean + s.d. of three independent determinations. ${ }^{\star} P<0.05$ (vs control cells with mock treatment). ${ }^{\#} P<0.05$ (vs control cells with DHT treatment). (B) Co-precipitation of AR and Sgl. Cell lysates $(500 \mu \mathrm{g})$ from PC3 transfected with pSG5-AR $(7 \mu \mathrm{g})$ and pSG5-Sgl $(7 \mu \mathrm{g})$ and cultured in medium supplemented with $10 \%$ normal FBS in the presence of $300 \mu \mathrm{m}$ zinc were incubated with an anti-AR polyclonal antibody $(2 \mu \mathrm{g})$. Input protein ( $25 \mu \mathrm{g}$ of total lysates) and the precipitated proteins were resolved on a $10 \%$ SDS-PAGE and blotted with an anti-Sgl or anti-AR antibody.

containing AR-binding sequences (e.g. LxxLL $v s$ LxxAA as a control) were transfected, and cell growth was monitored by MTT assay. In the presence of zinc, cell proliferation of both LNCaP and C4-2 was significantly inhibited by the wild-type peptide, but not the mutant peptide (Figure 3A). However, both wild-type and mutant peptides showed no significant inhibitory effects on their growth without zinc treatment (Figure 3B).

The expression levels of PSA, an androgen-inducible AR target and the currently most useful tumour marker, represent prostate cancer progression. Western blotting was then performed in LNCaP/C4-2 cells transfected with SgI peptides. In both lines cultured in the presence of zinc, PSA expression was considerably suppressed by transfection of the wild-type peptide, but not the mutant peptide (Figure 3C).

\section{DISCUSSION}

While functions of semenogelins have been thoroughly characterised in physiological environment especially in the male reproductive system, their roles in the development and progression of human malignancies remain poorly understood. We have 

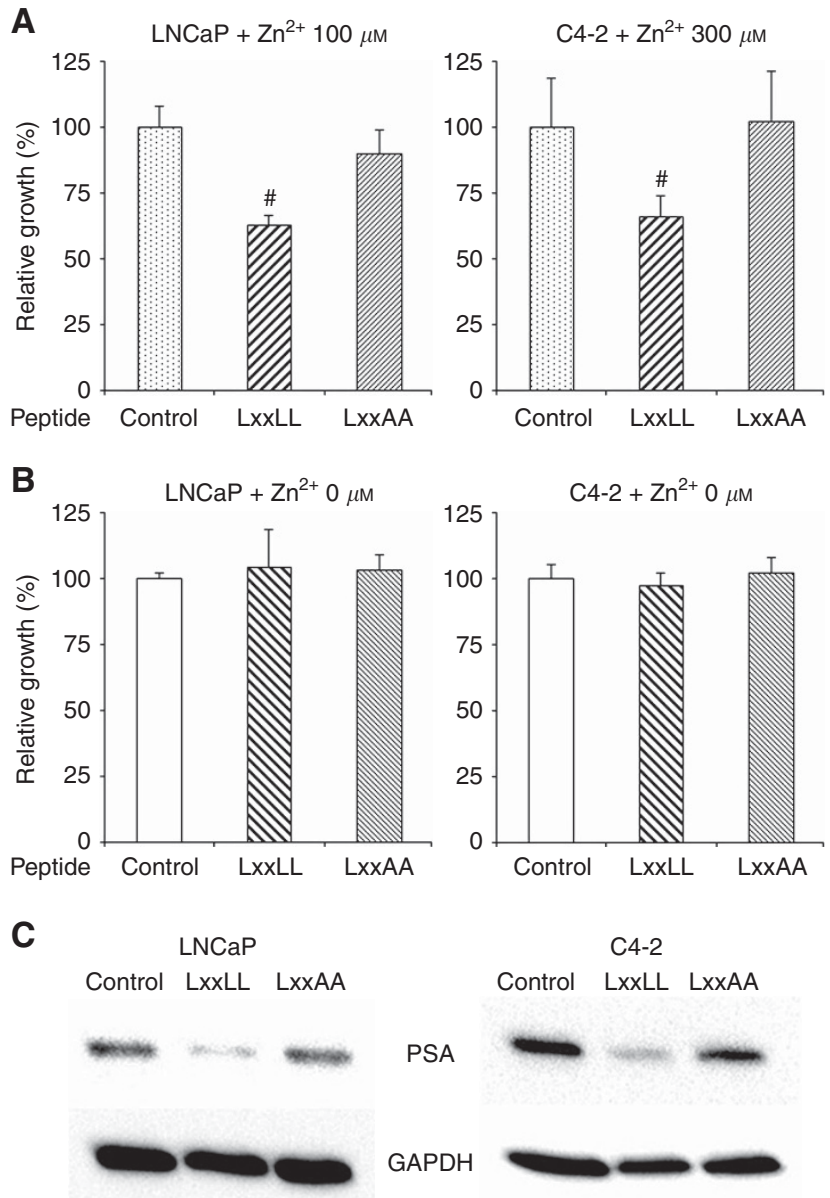

Figure 3. The effects of wild-type (WT) vs mutant (MT) Sgl peptides on the growth of prostate cancer cells. LNCaP and C4-2 cells were transfected with Xfect control or a Sgl peptide (250 ng) and cultured in medium supplemented with $10 \%$ normal FBS in the presence (A) or absence (B) of $100 \mu \mathrm{m}(\mathrm{LNCaP}) / 300 \mu \mathrm{m}$ (C4-2) zinc for $72 \mathrm{~h}$. Cell viability assayed with MTT is presented relative to cell number in each control line (first lanes; set as 100\%). Each value represents the mean + s.d. of three independent determinations. ${ }^{\#} P<0.05$ (vs control cells). (C) Total proteins extracted from LNCaP or C4-2 cells transfected with Xfect control or a Sgl peptide $(2 \mu \mathrm{g})$ and cultured in medium supplemented with $10 \%$ normal FBS in the presence of $300 \mu \mathrm{m}$ zinc for $24 \mathrm{~h}$ were analysed on western blots, using an antibody to PSA ( $33 \mathrm{kDa}$ ) or GAPDH (37 kDa).

previously found androgen- and zinc-dependent AR-SgI interactions in prostate cancer cells, which enhances androgen-mediated $\mathrm{AR}$ transcriptional activity (Ishiguro et al, 2015). This led to significant increases in the growth of prostate cancer cells and/or protection of the AR-positive cells from the cytotoxic effect of zinc (Canacci et al, 2011; Ishiguro et al, 2015), at least partially explaining why prostate cancer tissues contain relatively high levels of zinc (Costello and Franklin, 2006). In the present study, we further demonstrated that the LxxLL motif present in SgI protein played a critical role in the interactions with AR and that a peptide containing the motif inhibited the cell growth of both androgensensitive prostate cancer and CRPC.

We first confirmed that the wild-type SgI induced the proliferation of AR-positive prostate cancer cells only in the presence of zinc otherwise protected these cells against zinc cytotoxicity, and further showed a stimulatory effect on their migration. By contrast, a mutant SgI replacing the LxxLL with LxxAA did not significantly affect cell proliferation and migration in the presence of AR/androgens and zinc. Furthermore, the mutant SgI failed to not only interact with AR in prostate cancer cells but also significantly induce AR transactivation. These observations indicate that the LxxLL motif is essential and sufficient for mediating specific interactions between AR and SgI, as shown in other AR coregulators (Heery et al, 1997; van de Wijngaart et al, 2012). We therefore anticipated that interruption of AR-SgI interactions via the LxxLL motif resulted in considerable decreases in AR activity as well as tumour growth.

As aforementioned, AR signals remain critical in most of prostate cancer cells from patients with clinically defined CRPC (Coutinho et al, 2016). As expected, a SgI peptide containing the LxxLL motif was found to inhibit the proliferation and PSA expression similarly in androgen-sensitive LNCaP and castrationresistant C4-2 cell lines, presumably via interruption of interactions between $\mathrm{AR}$ and endogenous SgI, while $\mathrm{AR}-\mathrm{SgI}$ peptide interactions were not confirmed. Our findings thus provide a novel therapeutic target for prostate cancer and further suggest that peptide antagonists that interrupt AR-SgI interactions could successfully inhibit the growth of CRPC. However, further in vivo studies are necessary to determine the clinical relevance of our in vitro findings and address the feasibility of future therapeutic intervention. In particular, it remains difficult to deliver macromolecular drugs into cells even in animal models (Kurrikoff et al, 2016). It may also be required to determine whether the use of the short recombinant peptides derived for blocking AR-SgI interaction causes favorable or unfavorable outcome due to, for instance, off-target effects on AR interaction with other coregulators, especially those interacting with $A R$ via the LxxLL motif, and peptide conformational changes.

\section{ACKNOWLEDGEMENTS}

This work was supported by Department of Defense Prostate Cancer Research Program (W81XWH-13-1-0412) to HM.

\section{CONFLICT OF INTEREST}

The authors declare no conflict of interest.

\section{REFERENCES}

Canacci AM, Izumi K, Zheng Y, Gordetsky J, Yao JL, Miyamoto H (2011) Expression of semenogelins I and II and its prognostic significance in human prostate cancer. Prostate 71: 1108-1114.

Costello LC, Franklin RB (2006) The clinical relevance of the metabolism of prostate cancer; zinc and tumor suppression: connecting the dots. Mol Cancer 5: 17.

Coutinho I, Day TK, Tilley WD, Selth LA (2016) Androgen receptor signaling in castration-resistant prostate cancer: a lesson in persistence. Endocr Relat Cancer 23: T179-T197.

Heery DM, Kalkhoven E, Hoare S, Parker MG (1997) A signature motif in transcriptional co-activators mediates binding to nuclear receptor. Nature 387: 733-736.

Ishiguro H, Izumi K, Kashiwagi E, Zheng Y, Li Y, Kawahara T, Miyamoto H (2015) Semenogelin I promotes prostate cancer growth via functioning as an androgen receptor coactivator and protecting against zinc cytotoxicity. Am J Cancer Res 5: 738-747.

Izumi K, Li Y, Zheng Y, Gordetsky J, Yao JL, Miyamoto H (2012) Seminal plasma proteins in prostatic carcinoma: increased nuclear semenogelin I expression is a predictor of biochemical recurrence after radical prostatectomy. Hum Pathol 43: 1991-2000.

Jonsson M, Lundwall $\AA$, Malm J (2006) The semenogelins: proteins with functions beyond reproduction? Cell Mol Life Sci 63: 2886-2888. 
Kurrikoff K, Gestin M, Langel Ü (2016) Recent in vivo advances in cellpenetrating peptide-assisted drug delivery. Expert Opin Drug Deliv 13: 373-387.

Liang JY, Liu YY, Zou J, Franklin RB, Costello LC, Feng P (1999) Inhibitory effect of zinc on human prostatic carcinoma cell growth. Prostate 40: 200-207.

Lilja H, Oldbring J, Rannevik G, Laurell CB (1987) Seminal vesicle-secreted proteins and their reactions during gelation and liquefaction of human semen. J Clin Invest 80: 281-285.

Miyamoto H, Marwah P, Marwah A, Yang Z, Chung C-Y, Altuwaijri S, Chang C, Lardy H (2005) Identification of steroid derivatives that function as potent antiandrogens. Int J Cancer 117: 866-872.

Torre LA, Bray F, Siegel RL, Ferlay J, Lortet-Tieulent J, Jemal A (2015) Global cancer statistics, 2012. CA Cancer J Clin 65: 87-108.

Untergasser G, Rumpold H, Plas E, Witkowski M, Pfister G, Berger P (2000) High levels of zinc ions induce loss of mitochondrial potential and degradation of antiapoptotic Bcl-2 protein in in vitro cultivated human prostate epithelial cells. Biochem Biophys Res Commun 279: 607-614.

van de Wijngaart DJ, Dubbink HJ, van Royen ME, Trapman J, Jenster G (2012) Androgen receptor coregulators: recruitment via the coactivator binding groove. Mol Cell Endocrinol 352: 57-69.

Zheng Y, Ishiguro H, Ide H, Inoue S, Kashiwagi E, Kawahara T, Jalalizadeh M, Reis LO, Miyamoto H (2015) Compound A inhibits bladder cancer growth predominantly via glucocorticoid receptor transrepression. Mol Endocrinol 29: 1486-1497.

This work is published under the standard license to publish agreement. After 12 months the work will become freely available and the license terms will switch to a Creative Commons AttributionNonCommercial-Share Alike 4.0 Unported License. 